American Journal of Applied Sciences 5 (11): 1552-1557, 2008

ISSN 1546-9239

(C) 2008 Science Publications

\title{
A Novel Thermooptic Polymer Switch Based on Directional Coupler Structure
}

\author{
Abu Sahmah Mohd Supa'at, Mohd. Haniff Ibrahim, Abu Bakar Mohammad, \\ Norazan Mohd Kassim and Nurzal Effiyana Ghazali \\ Photonics Technology Centre, Faculty of Electrical Engineering, Universiti Teknologi Malaysia \\ 81310 UTM Skudai, Johor Darul Takzim, Malaysia
}

\begin{abstract}
A novel thermooptic (TO) polymer switch based on directional coupler structure is proposed for the first time. The key element of the proposed switch is a buried square core polymer waveguides which has been designed for optimum mode matching and coupling with the single mode optical fiber configuration and have large effective index difference by the thermooptic effects. At a wavelength of $1.55 \mu \mathrm{m}$ with the index contrast of 0.005 between the core and the cladding, the crosstalk of $-40 \mathrm{~dB}$ and $-37 \mathrm{~dB}$ for the cross-state and the bar-state of TO switch is demonstrated, respectively.
\end{abstract}

Key words: Optical polymer, optical waveguide, thermooptic switch, directional coupler

\section{INTRODUCTION}

There is an increasing need for optical switch matrices for routing, switching, protection switching, cross connection and add-drop multiplexing. The recent progress in wavelength division multiplex (WDM) lightwave communication system will further increase the necessity of optical switch modules. Several optical switch architectures have been proposed and implemented in silica on silicon or glass ${ }^{[1]}$, Ti$\mathrm{LiNbO}_{3}{ }^{[2]}$, semiconductors ${ }^{[3]}$ and polymers. Polymeric materials are particularly attractive in integrated optics because of their ability to be processed rapidly, costeffectively and with high yields ${ }^{[4]}$ such as demonstrated in the development of high-speed Mach-Zehnder (MZ) modulator ${ }^{[6]}$ and polarization control devices ${ }^{[7]}$. Classes of polymers used in integrated optics include acrylates, polyimides, polycarbonates and olefin (e.g., cyclobutene). Ironically, the $2 \times 2$ polymeric switches have not been intensively investigated up to now and there are only a few reports on the devices based on the directional couplers (DC) and the balanced bridge MZ interferometer switch incorporating 3-dB couplers ${ }^{[8]}$.

In this paper, a novel development of $2 \times 2$ directional coupler thermooptic polymer switch (DCTOPS), specifically using of all thermooptic (TO) polymer material is presented. The ultraviolet (UV) curable acrylate adhesive polymers have been chosen as the switch material due to advantages such as ease of fabrication, integration feasibility and suitable for mass- production. The advantage of investigating a $2 \times 2$ DCTOPS is that it can perform a number of useful functions in thin film devices. Additionally, the DCTOPS uses less switching power and small in size, which implies low propagation loss and insertion loss, which is suitable for large switching matrices devices for optical cross connect (OXC) applications ${ }^{[9]}$.

\section{SWITCH STRUCTURE AND PRINCIPLE}

The directional coupler consists of two monomodal waveguide channels with identical width and effective index, running parallel to each other in a central section. In this central section, the structure has mirror symmetry with respect to the axis a-b. In that way symmetric and asymmetric field are obtained as shown in Fig. $1^{[12]}$.

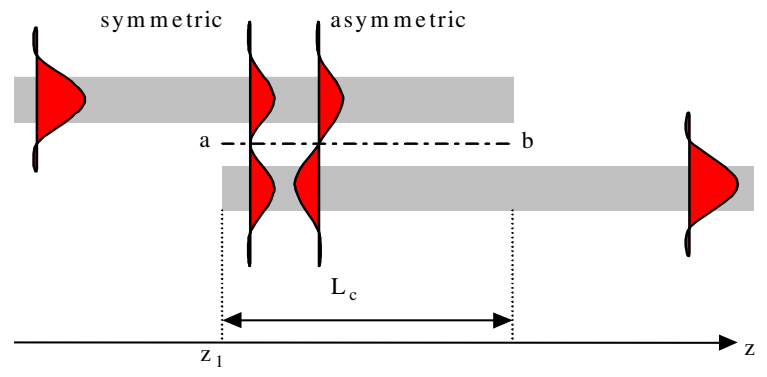

Fig. 1: Structure consisting of two three-layer sections and one symmetrical five-layer sections

Corresponding Author: Abu Sahmah Mohd Supa'at, Photonics Technology Centre, Faculty of Electrical Engineering, Universiti Teknologi Malaysia 81310 UTM Skudai, Johor Darul Takzim, Malaysia Tel: 607-5535205, 607-5566272 
At $\mathrm{z}=\mathrm{z}_{1}$ the power of the incoming channel mode is divided equally between both system modes. Note that in the middle section $\mathrm{z}=\mathrm{z}_{1}$ all power is in the upper channel and no power in the lower one. This can be seen directly by adding the fields, taking into account that the phase differences between both modes are still zero. During propagation this phase difference will change however because the modes have different propagation constants $\beta_{\text {sym }}$ and $\beta_{\text {asym }}$ respectively. After a distance

$$
\Delta \mathrm{z}=\frac{\pi}{\left(\beta_{\mathrm{sym}}-\beta_{\mathrm{asym}}\right)}
$$

the phase difference between the modes is equal to $\pi$. By adding again the modal and taking into account this $\pi$ phase difference, it can be directly seen that now all power is in the lower channel and no power left in the upper one. This specific length is called the coupling length, $\mathrm{L}_{\mathrm{c}}$ and in this specific system, this coupling length is given by

$$
\mathrm{L}_{\mathrm{c}}=\frac{\pi}{\left(\beta_{\mathrm{sym}}-\beta_{\mathrm{asym}}\right)}
$$

Eq. (2) can be written in the form of the two lowest order modes as symmetric and asymmetric with the effective indices $N_{\text {sym }}$ and $N_{\text {asym }}$ respectively.

$$
\mathrm{L}_{\mathrm{c}}=\frac{\lambda}{2\left(\mathrm{~N}_{\mathrm{sym}}-\mathrm{N}_{\mathrm{asym}}\right)}
$$

where $\lambda$ is the optical wavelength in free space.

Figure 2 shows a vertical cross section of $2 \times 2$ DCTOPS with the design parameters shown. The structure consists of two symmetric waveguides with the dimension $w \times t$, that are close together but separated by a waveguide spacing of $\mathrm{g}$. $\mathrm{D}$ is the heater electrode thickness, $\mathrm{B}$ is the heater width and $\mathrm{d}$ is the center-tocenter distance between the core and the heater electrode. The thickness of the upper cladding and the lower cladding are limited by mode attenuation due to the metal heater electrode and the substrate respectively. The thickness of the upper cladding is bound to attenuation into the heater electrode while the lower cladding is bound to attenuation into the substrate $^{[13]}$. To avoid this attenuation, the cladding has been taken rather thick and therefore the substrate layer does not have to be included in the optical simulation. Bend waveguides with curvature radius of $R_{c}$ are connected to both the input and output ports to form the $2 \times 2$ DCTOPS for practical implementation with single mode optical fiber.

\section{THERMAL ANALYSIS AND TEMPERATURE PROFILE}

In general we can distinguish three different types of heat transport mechanism namely convection, radiation and conduction. However, due to negligible contribution of radiation and convection mechanisms ${ }^{[14]}$, heat conduction is the only considered mechanism, which will be accounted on the influence of temperature profiles in the optical waveguides, for which Wang et al., ${ }^{[13]}$ and Kokkas ${ }^{[16]}$ also used this approach. Mathematically the heat equation can be stated as ${ }^{[15]}$ :

$$
\nabla^{2} \mathrm{~T}-\frac{1}{\alpha} \frac{\partial \mathrm{T}}{\partial \mathrm{t}}=-\frac{\mathrm{Q}_{\mathrm{gen}}}{\mathrm{k}}
$$

where $\mathrm{T}$ is the temperature, $\mathrm{Q}_{\mathrm{gen}}$ is the power generated per unit volume, $\mathrm{k}$ is the thermal conductivity of the material and $\alpha$ is the thermal diffusivity. In this problem we will seek solutions for the temperature profile in two-dimensional region of Fig. 2 with specified boundary conditions. This can be done as the dimension of the heater in the direction of propagation is in the order of millimeters and the other dimensions are in the order of microns. Considering the steady state heat conduction, Eq. 4 can be deduced to

$$
\nabla^{2} \mathrm{~T}+\frac{\mathrm{Q}_{\mathrm{gen}}}{\mathrm{k}}=0
$$

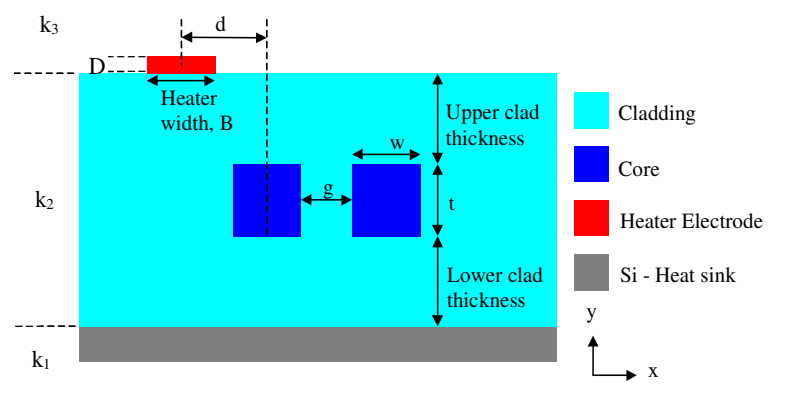

Fig. 2: Vertical cross-section of $2 \times 2$ DCTOPS. Material thermal conductivities, $\mathrm{k}_{1}(\mathrm{Si})=150, \mathrm{k}_{2}$ $($ Polymers $)=0.2$ and $\mathrm{k}_{3}($ air $)=2.6 \times 10^{-2} \mathrm{~W} / \mathrm{m}^{\circ} \mathrm{C}$. All dimensions are in $\mu \mathrm{m}$ 


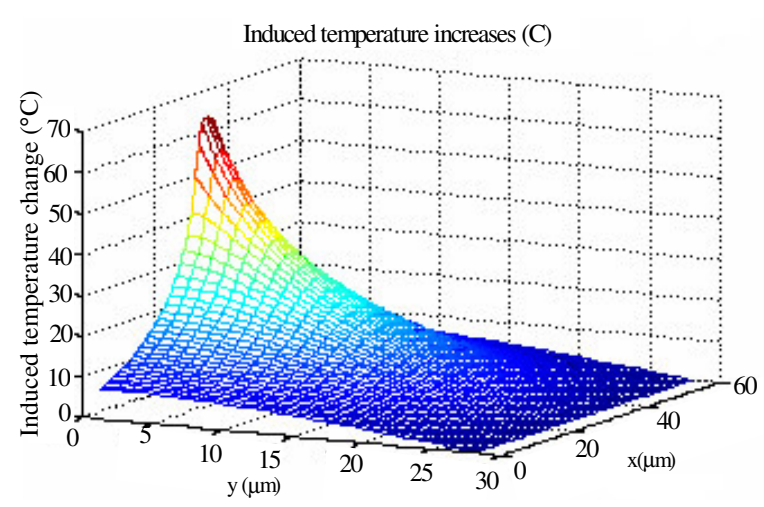

Fig. 3: Induced temperature profile across the structure shown in Fig. 1 with the total thickness, $\mathrm{t}=27$ $\mu \mathrm{m}, \mathrm{B}=7 \mu \mathrm{m}$ and $\mathrm{P} / \mathrm{L}=20 \mathrm{~mW} / \mathrm{mm}$

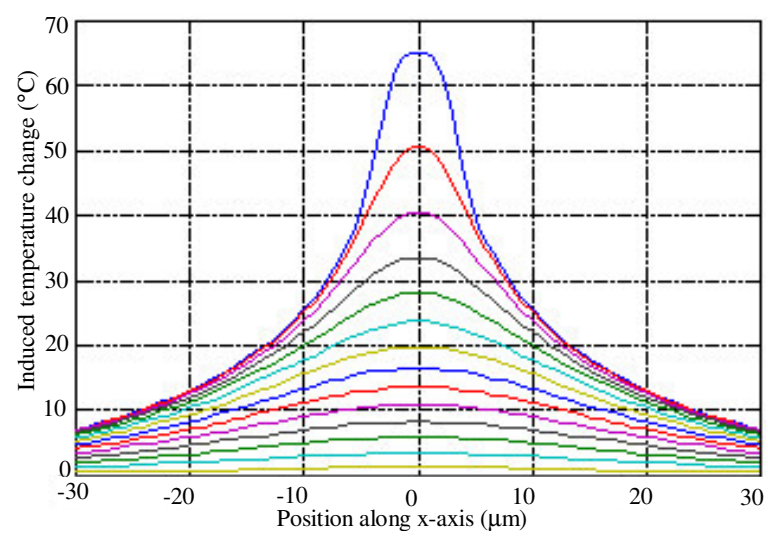

Fig. 4: Temperature profile in the waveguide for varying distance under the heater electrode of $\Delta \mathrm{y}=2 \mu \mathrm{m}$ and $\mathrm{L}=1 \mathrm{~mm}$

the induced temperature distributions across the buried square core (BSC) waveguides cross-section, induced by $7 \mu \mathrm{m}$ wide thin film heater with the total thickness of the structure of $27 \mu \mathrm{m}$ is shown in Fig. 3.

The core and the cladding layers are assumed to have the same thermal conductivity. As previously mentioned, the substrate can be considered as a perfect heat sink. The maximum temperature at the metallic thin film heater and the cladding top surface interface is around $65^{\circ} \mathrm{C}$. It can be seen that the lateral width of temperature profile distribution at the top cladding surface is smaller than the bottom surface. This indicates that we can control the temperature profile so as the resulting phase shift of TO induce waveguide by properly choosing the upper cladding thickness for BSC waveguide. The temperature profile in the waveguide for varying distance under the thin film heater has been plotted as shown in Fig. 4.

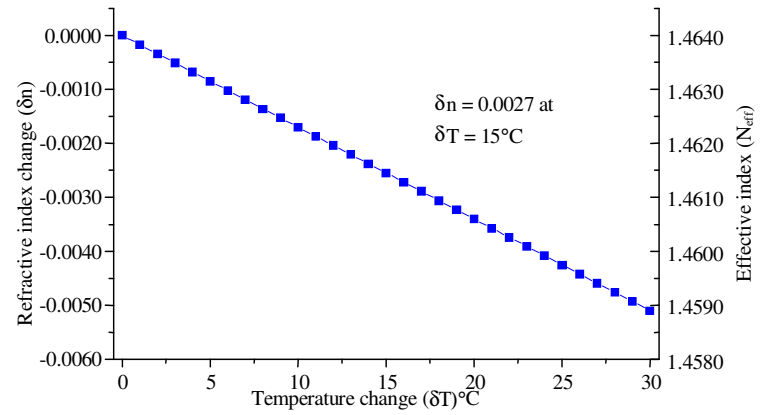

Fig. 5: Refractive index change in TO waveguide as a function of temperature change due to thermal induced temperature in the thin film heater

As shown in the Fig. 4 was 14 values of lateral temperature profile for $\Delta \mathrm{y}=2 \mu \mathrm{m}(\mathrm{y}=0, \mathrm{y}=\mathrm{t})$. From this figure, it can be concluded the temperature profile meets the boundary conditions of the given structure. The numerical results have been checked with a simple analytical solution ${ }^{[13]}$ and the results are in good agreement with each other. These also show that the temperature distribution when the thin film heater is positioned asymmetrically from the first waveguide to be heated, thermal coupling will decreases tremendously to the second adjacent waveguide. With $\mathrm{d}$ $=5 \mu \mathrm{m}$, that is the spacing of thin film heater to the first waveguide to be heated, the resulting phase shift or refractive index change has been calculated based on the induced temperature distribution shown in Fig. 4 is given in Fig. 5.

\section{RESULTS AND DISCUSSION}

The symmetric, $\mathrm{N}_{\mathrm{sym}}$ and asymmetric, $\mathrm{N}_{\mathrm{asym}}$ modes have to be determined to calculate the coupling length $\mathrm{L}_{\mathrm{c}}$ of two identical waveguides close to each other. At larger distance the two system modes that can propagate in the couple waveguides structure will have the same propagation constant because the fundamental system mode is split into two fundamental channel modes. The simulation has been performed to calculate the two lowest order system modes while varying the waveguides spacing, g. The result obtained is shown in Fig. 6.

Figure 6 shows the effective indices of these modes $\mathrm{N}_{\text {sym }}$ and $\mathrm{N}_{\text {asym }}$ as a function of waveguides spacing. It can be seen after the waveguides spacing, $\mathrm{g}=12 \mu \mathrm{m}$, the two lowest order modes have the same effective index. The coupling length is then calculated as shown in Fig. 7.

From the graph with the waveguide spacing of 5 $\mu \mathrm{m}$, it can be shown that the corresponding coupling length is about $2500 \mu \mathrm{m}$. 


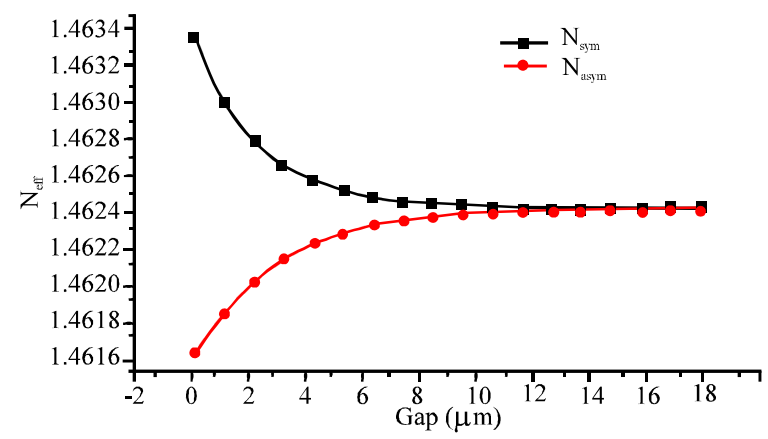

Fig. 6: Effective indices, $\mathrm{N}_{\mathrm{eff}}$ as a function of waveguide gap

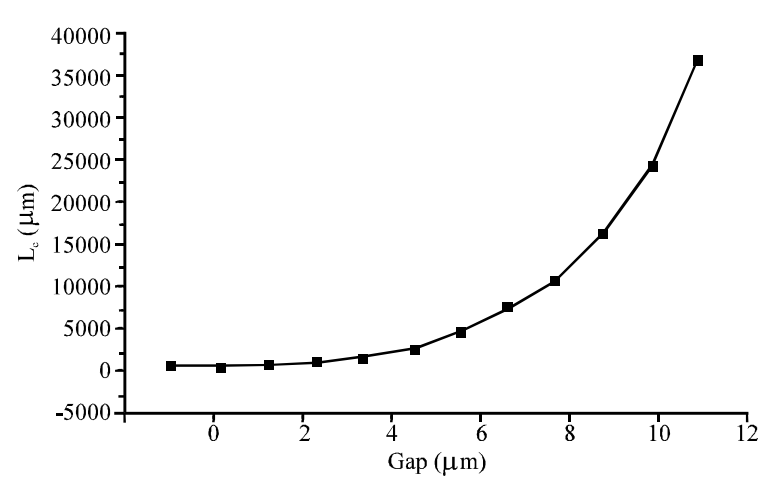

Fig. 7: Coupling length, $\mathrm{L}_{c}$ as a function of waveguide gap

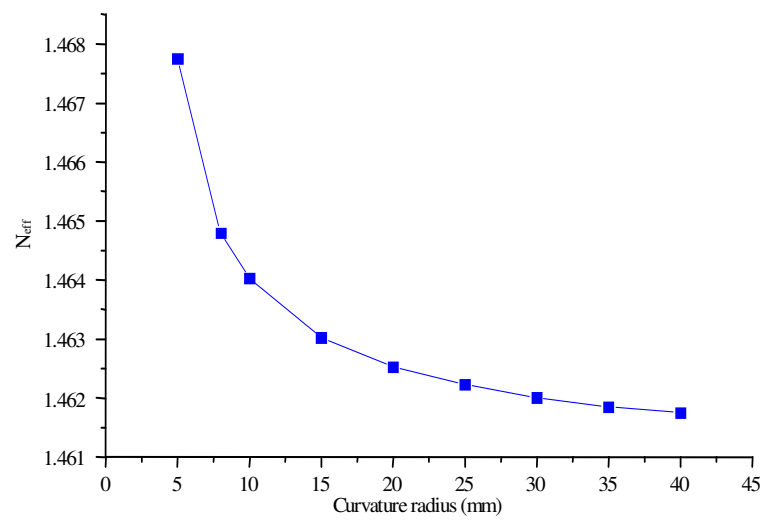

Figure 8: Mode effective indices as a function of the curvature radius, $R_{c}$

For the bend waveguide the simulation has been performed using three dimensional scalar finite difference and transverse electric polarization. The graph has been plotted for the modal indices, $\mathrm{N}_{\mathrm{eff}}$ of the upper bound refractive index of the core as a function of curvature radius $R_{c}$ as shown in Fig. 8 .
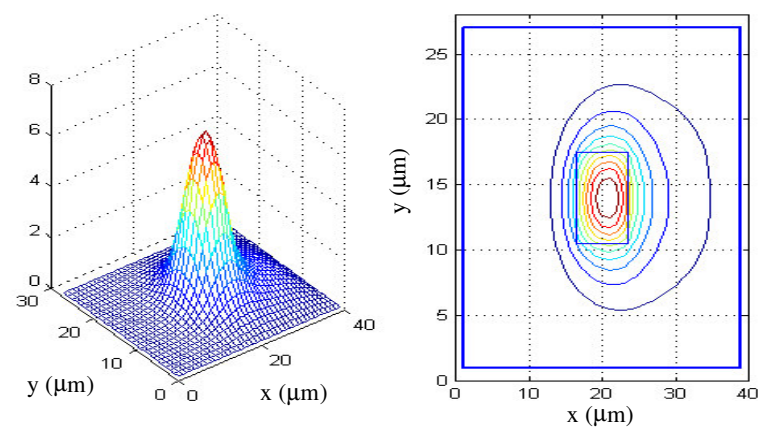

(a) $\mathrm{R}_{\mathrm{c}}=8000 \mu \mathrm{m}$
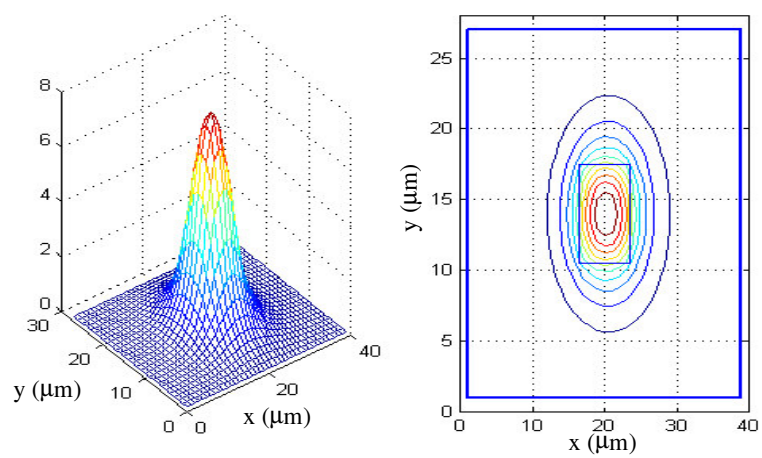

(b) $\mathrm{R}_{\mathrm{c}}=25000 \mu \mathrm{m}$

Fig. 9: Examples of three dimensional and contour plot of light intensity distribution for bend waveguides of curvature radius (a) $R_{c}=8000$ $\mu \mathrm{m}$ and (b) $R_{c}=25000 \mu \mathrm{m}$, respectively. z-axis is arbitrary unit

As can be seen from the graph, as the curvature radius increases the modal indices of the bend waveguide decreases to the lower bound of the vicinity of the cladding refractive index. The examples of three dimensional and contour plot of light intensity distribution within the BSC bend waveguide structure are shown in Fig. 9 for radius of curvature (a) $R_{c}=$ $8000 \mu \mathrm{m}$ and (b) $25000 \mu \mathrm{m}$, respectively.

For the device length, the values of the offset have been chosen as 119 and $200 \mu \mathrm{m}$ for practical design of the directional coupler and the radius of the bend has been varied from 1 to $30 \mathrm{~mm}$. This function has been plotted as shown in Fig. 10.

As expected it can be seen that the smaller the bend radius and the offset the shorter the device length will be. However, for the design of the S-bend, the radius of curvature greater than $20 \mathrm{~mm}$ so that the loss of the bend waveguides is negligible and the device length of $3 \mathrm{~mm}$, had been chosen.

Finally the normalized power as a function of distance for $2 \times 2$ DCTOPS has been simulated. The 
Am. J. Applied Sci., 5 (11): 1552-1557, 2008

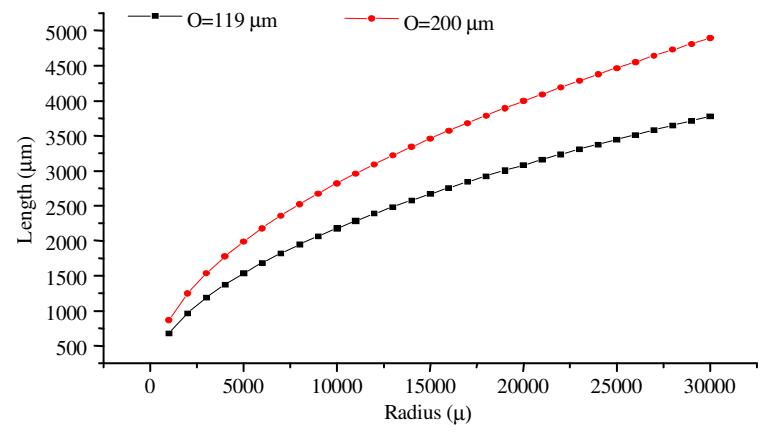

Fig. 10: Device length, $\mathrm{L}$ as a function of bend radius, $\mathrm{R}_{\mathrm{c}}$ for an Offset, $\mathrm{O}=119 \mu \mathrm{m}$ and $200 \mu \mathrm{m}$, respectively

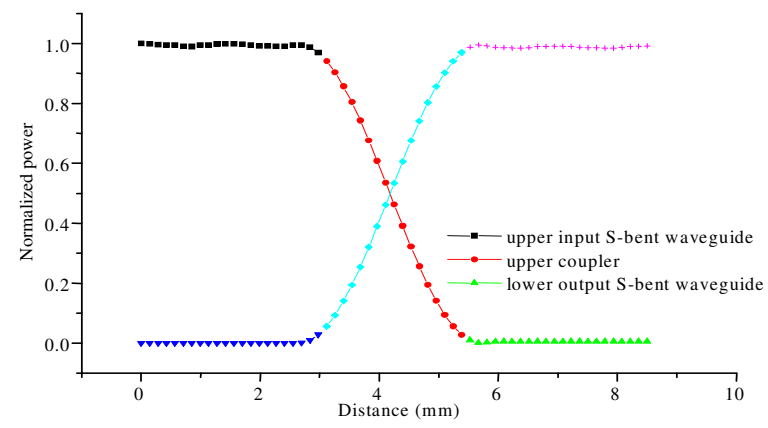

Fig. 11: Normalized power as a function of distance for $2 \times 2$ DCTOPS (cross-state) with the waveguides separation of the input and output end of $250 \mu \mathrm{m}$ and the $\mathrm{O}=119 \mu \mathrm{m}$

meant value of refractive index change was taken to be 0.0027 due to the induced temperature change in TO waveguide of $15^{\circ} \mathrm{C}$. The waveguides end separation of $250 \mu \mathrm{m}$ and the offset of $119 \mu \mathrm{m}$ had been taken for practical implementation. The results obtained are shown in Fig. 11 for the cross-state while Fig. 12 for the bar-state.

From these data the crosstalk in $\mathrm{dB}$ for the two states, which are the cross-state and the bar-state have been calculated. The crosstalk in $2 \times 2$ DCTOPS is defined as the ratio of light power in the undesired output port to the power in the desired output port. From Fig. 11, the crosstalk value is $-40 \mathrm{~dB}$ for the cross-state had been achieved. From Fig. 12, there was ripple in the lower and upper couplers for the bar state. This could be expected due to the effect of refractive index change as a result of induced temperature change. Based on the calculation, the crosstalk value is $-37 \mathrm{~dB}$ which is slightly higher than in the cross-state. However, this value could be increased, once the fabricated switch is realized since at the bar-state the

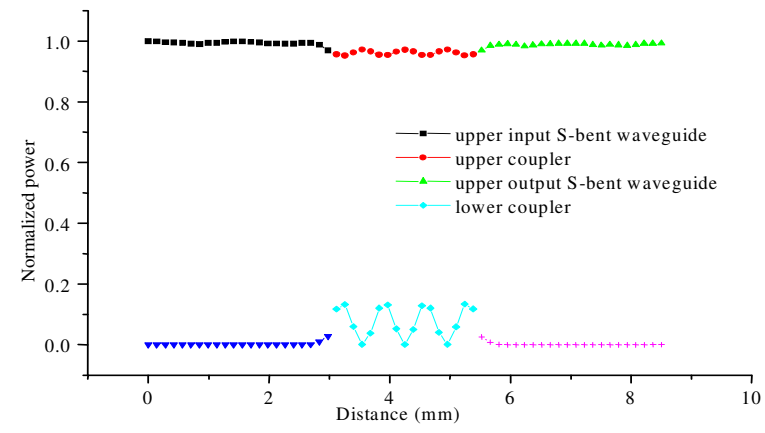

Fig. 12: Normalized power as a function of distance for $2 \times 2$ DCTOPS (bar-state) with the waveguides separation of the input and output end of 250 $\mu \mathrm{m}$ and the $\mathrm{O}=119 \mu \mathrm{m}$

induced temperature change can be controlled. Therefore the crosstalk value less than $-40 \mathrm{~dB}$ could be expected.

\section{CONCLUSIONS}

The design to optical switch presented in this paper shows that a directional coupler based of BSC waveguide in polymer materials appears feasible. The coupling length of $2500 \mu \mathrm{m}$, waveguide spacing of 5 $\mu \mathrm{m}$ and the geometry of the cross section of the structure given above can be used as a design parameter for fabrication of TO optical switch. The thermal analysis of the temperature distribution in the BSC polymer waveguides was calculated and the model showed good agreement with other researcher. At a wavelength of $1.55 \mu \mathrm{m}$ with the index contrast of 0.005 between the core and the cladding, the crosstalk of -40 $\mathrm{dB}$ and $-37 \mathrm{~dB}$ for the cross-state and the bar-state of TO switch is possible, respectively.

\section{ACKNOWLEDGEMENT}

The authors would like to thank the Ministry of Science, Technology and Innovation (MOSTI) for financing this project. Our gratitude also goes to the administration of Universiti Teknologi Malaysia (UTM) especially the Research Management Center (RMC) for their support and all members of Photonics Technology Centre.

\section{REFERENCES}

1. Shibata, T., 2002. High performance optical switches integrated with silica-based planar lightwave circuits on Si. Lasers and Electro-Optics Society (LEOS'02), 2: November 10-14. 730-731. 
2. Krahenbuhl, R., M.M. Howerton, J. Dubinger and A.S. Greenblatt, 2002. Performance and modeling of advanced Ti: $\mathrm{LiNbO}_{3}$ digital optical switches. J. Lightwave Technol., 20 (1): 92-99.

3. Hamamoto, K., T. Sasaki, T. Matsumoto and K. Komatsu, 1996. Insertion loss free $1 \times 4$ optical switch fabricated using bandgap-energy-controlled selective MOVPE. Elect. Lett., 32 (24): 2265-2266.

4. Eldada, L. and L.W. Shacklette, 2000. Advances in polymer integrated optics. IEEE J. Selected Topics in Quantum Elect., 6 (1): 54-68.

5. Pun, E.Y.B. and W.H. Wong, 2002. Optical polymeric waveguides and devices. Proceedings of IEEE/LEOS Workshop on Fibre and Optical Passive Components. June 5-6: 126-132.

6. Spickermann, R., M.G. Peters and N. Dagli, 1996. A polarization independent GaAs-AlGaAs electrooptic modulator. J. Quantum Elect., 32 (5): 764-769.

7. Oh, M.C., W.Y. Hwang and J.J. Kim, 1997. Integrated optic polarization controlling devices using electro-optic polymers. ETRI J., 18 (4): 287-299.

8. Oh, M.C., W.Y. Hwang, H.M. Lee, S.G. Han and Y.H. Won, 1997b. Electrooptic polymer modulators operating in both TE and TM modes incorporating a vertically tapered cladding. IEEE Photonics Technol. Lett., 9 (9): 1232-1234.

9. Kozhevnikov, M., N.R. Basavanhally, J.D. Weld, Y.L. Low, P. Kolodner, C.A. Bolle, R. Ryf, A.R. Papazian, A. Olkhoverts, F. Pardo, J. Kim, D.T. Neilson, V.A. Aksyuk and J.V. Gates, 2003. Compact $64 \times 64$ micromechanical optical cross connect. IEEE Photonics Technol. Lett., 15 (7): 993-995.
10. Diemeer, M.B.J., 1998. Polymeric thermo-optic space switches for optical communications. J. Optical Mate., 9: 192-200.

11. Supa'at, A.S.M., A.B. Mohammad and N.M. Kassim, 2002a. Modelling techniques for rectangular dielectric waveguides-ribs waveguides. Jurnal Teknologi, 36 (D): 129-143.

12. Ridder, R.M., 2002. Theory optical basic functions and Microsystems: Application notes. LDG, University of Twente. Enschede, The Netherlands.

13. Wang, W.K., H.J. Lee and P.J. Anthony, 1996. Planar silica-glass optical waveguides with thermally induced lateral mode confinement. J. Lightwave Technol., 14 (3): 429-436.

14. Veldhuis, G.J., 1998. Bent waveguide devices and mechano-optical switches. University of Twente. Enschede, The Netherlands: Ph.D Thesis.

15. Zemansky, M.W. and R.H. Dittman, 1983. Heat and Thermodynamics. Mc Graw Hill Book Company.

16. Kokkas, A.G., 1974. Thermal analysis of multiple layer structures. IEEE Trans. Elect. Devices, 21 (11): 675-681.

17. Nakagawa, G., T. Yamamoto, S. Sasaki, M. Norimatsu, N. Yamamoto, T. Nosaka, K. Terada, K. Tanaka, K. Miura and M. Yano, 1998. High power and high sensitivity planar lightwave circuit module incorporating a novel passive alignment method. J. Lightwave Technol., 16 (1): 66-72.

18. Lytel, R., 1994. EO polymer materials and devices: From research to reality. IEEE Nonlinear Optic: Material, Fundamental and Applications (NLO' 94). July 25-29: 3-5. 